\title{
Polycomb chromobox 4 enhances migration and pulmonary metastasis of hepatocellular carcinoma cell line MHCC97L
}

\author{
MEI Zhu ${ }^{1}$, JIAO HuiKe ${ }^{2}$, WANG Wei ${ }^{1}$, LI Jie $^{1}$, CHEN GuoQiang ${ }^{1,2}$ \& XU Ying ${ }^{2 *}$ \\ ${ }^{1}$ Department of Pathophysiology, Key Laboratory of Cell Differentiation and Apoptosis of Ministry of Education; Ruijin Hospital, Shanghai \\ Jiao Tong University School of Medicine, Shanghai 200025, China; \\ ${ }^{2}$ Institute of Health Sciences, Shanghai Institutes for Biological Sciences of Chinese Academy of Sciences \& Shanghai Jiao Tong University \\ School of Medicine, Shanghai 200025, China
}

Received March 22, 2014; accepted April 3, 2014; published online May 15, 2014

\begin{abstract}
We recently report that the expression of polycomb chromobox $4(\mathrm{Cbx} 4)$ is significantly correlated with the overall survival of a great cohort of hepatocellular carcinoma (HCC) patients and it enhances hypoxia-induced vascular endothelial growth factor (VEGF) expression and angiogenesis in HCC cells through enhancing sumoylation of hypoxia inducible factor-1alpha (HIF-1 $\alpha$ ). Here we continue to investigate the potential effects of $\mathrm{Cbx} 4$ on the migration and metastasis of the metastatic HCC cell line MHCC97L. Our results show that Cbx4 overexpression in the cell line increases the in vitro vessel formation of vascular endothelial cells in its SUMO interaction motifs-dependent manner, and promotes the in vitro migration of the cancer cell, which can be effectively abrogated by anti-VEGF antibody. Although Cbx4 expression does not impact the in vitro growth of MHCC97L cells, it still promotes the progression and metastasis of orthotopically transplanted tumors in nude mice. These results further support the role of $\mathrm{Cbx} 4$ as a SUMO E3 ligase in the progression and metastasis of HCC.
\end{abstract}

polycomb chromobox 4 , hepatocellular carcinoma, vascular endothelial growth factor, metastasis

Citation: Mei Z, Jiao HK, Wang W, Li J, Chen GQ, Xu Y. Polycomb chromobox 4 enhances migration and pulmonary metastasis of hepatocellular carcinoma cell line MHCC97L. Sci China Life Sci, 2014, 57: 610-617, doi: 10.1007/s11427-014-4663-9

Polycomb group proteins (PcG), a set of transcriptional repressors which impose the histone $\mathrm{H} 3$ lysine 27 tri-methylation for repression of gene expression [1,2], assemble into at least two major classes of multimeric protein polycomb repressive complexes (PRC) in mammal, that is, PRC1 and PRC2. They are essential for embryonic development and stem cell renewal and contribute widely to a series of biological processes such as cell cycle, DNA repair, cell differentiation, senescence and death [1,3-5]. Accordingly, the increasing lines of evidence demonstrate that dys-regulations of genes encoding PcG proteins, such as Bmi1, Cbx7 and Ezh2 are associated with tumorigenesis of many kinds of cancers [6-8].

*Corresponding author (email: yxu01@ sibs.ac.cn)
Like other members of the Cbx family, polycomb chromobox (Cbx) protein $4(\mathrm{Cbx} 4$, also designated as polycomb 2, Pc2) can bind to methylated histones as an important component for PRC1 [9]. However, $\mathrm{Cbx} 4$ but not other members of Cbx family has also been reported to act as the small ubiquitin-related modifier (SUMO) E3 ligase, which can enhance the sumoylation of transcriptional co-repressor $\mathrm{C}$ terminal binding protein (CtBP) [10], Smad-interacting protein 1 (SIP1) [11], DNA methyltransferase 3a (Dnmt3a) [12], multifunctional $\mathrm{Ca}^{2+}$-binding protein centrin-2 [13] and homeodomain-interacting protein kinase 2 (HIPK2) [14]. Structural analyses reveal that the N-terminal chromodomain and two SUMO interaction motifs (SIM1 and SIM2) appear to be required for its repressor and SUMO E3 ligase activity, respectively [15-18]. 
More recently, we reported that $\mathrm{Cbx} 4$ expression is significantly correlated with the overall survival of a great cohort of hepatocellular carcinoma (HCC) patients and of subcutaneously and orthotopically transplanted HCC mice [17]. Further, Cbx4 enhances hypoxia-induced vascular endothelial growth factor (VEGF) expression and angiogenesis in HCC cell lines such as SMMC-7721, HepG-2, BEL-7402 and MHCC97L [17]. Mechanically, Cbx4 enhances sumoylations of hypoxia inducible factor-1alpha $(\mathrm{HIF}-1 \alpha)$ protein, thus increasing the transcriptional activity of HIF-1 in its SUMO E3 ligase activity-dependent mechanisms $[17,18]$. Here we continue to investigate the potential effects of $\mathrm{Cbx} 4$ on the migration and metastasis of MHCC97L, a metastatic HCC cell line [19].

\section{Materials and methods}

\subsection{Cell culture and transfection}

293T cells were obtained from Cell Resource Center of Shanghai Institute for Biological Science, Chinese Academy of Sciences (Shanghai, China). Dr. Qing LunXiu at Fudan University (Shanghai, China) and Dr. Yi ZhengFang at East China Normal University (Shanghai, China) provided us MHCC97L [19] and human umbilical vascular endothelial cells (HUVEC) respectively. 293T cells and MHCC97L cells were cultured in DMEM containing $10 \%$ fetal bovine serum (FBS) and 1\% penicillin/streptomycin. HUVEC cells were maintained in ECM containing 10\% FBS. Hypoxic condition was performed in a specially designed hypoxic incubator (Thermo Electron, Forma, USA) with $1 \% \quad \mathrm{O}_{2}$, $94 \% \mathrm{~N}_{2}$ and $5 \% \mathrm{CO}_{2}$. Lipofectamine 2000 transfection reagent (Invitrogen, USA) was used for transient transfection. Transient co-transfection with helper plasmids was performed into 293T cells to prepare retroviruses. Cells were infected at approximately $70 \%$ density in DMEM supplemented with $8 \mu \mathrm{g} \mathrm{mL}^{-1}$ of polybrene. Twenty-four hours later, DMEM medium containing $10 \% \mathrm{FBS}$ and $1 \mu \mathrm{g} \mathrm{mL}$ puromycin was added to select stable cell lines, as we described previously [17].

\subsection{Plasmid construction}

Cbx4-expressing pCMV plasmids with amino-terminal Flag tag were generously provided by Dr. Weng JieMin at East China Normal University (Shanghai, China). Flag-tagged Cbx4 mutants were constructed by using QuikChange ${ }^{\mathrm{TM}}$ Lightning Site-Directed Mutagenesis Kit (Stratagene, USA). Flag-Cbx4 and its mutants were cloned into pBABE puro vector (Cell Biolabs, Inc., USA) for stable transfection as described previously [17].

\subsection{In vitro tube formation}

According to the manufacturer's instructions, the Matrigel was placed in a refrigerator at $4^{\circ} \mathrm{C}$ overnight to thaw. Once it had thawed, 24-well plates were coated with $100 \mu \mathrm{L} /$ well of Matrigel (BD Biosciences, USA) and immediately placed in an incubator at $37^{\circ} \mathrm{C}$ for $1 \mathrm{~h}$. HUVEC $\left(2 \times 10^{4}\right)$ in $1 \mathrm{~mL}$ of conditional DMEM medium, which had been incubated with $1.5 \times 10^{5}$ MHCC97L cells for $48 \mathrm{~h}$, were seeded onto the solidified gel. After incubating in an atmosphere of $5 \%$ $\mathrm{CO}_{2}$ and $95 \%$ air at $37^{\circ} \mathrm{C}$ for $16 \mathrm{~h}$ to allow the formation of a capillary-like structure, the endothelial tubes were counted under photomicroscope at $200 \times$ magnification over five fields in each slide.

\subsection{Immunohistological chemistry staining}

Sections were stained with anti-Cbx4 (dilution 1:25, SC19299, USA) and anti-VEGF polyclonal antibody (dilution 1:500, SC-152, USA) to analyze their protein levels. All responses were followed by staining with the corresponding HRP-conjugated secondary antibody (KIT-9719 and KIT9707, Maixin Biotechnology, China). Images were captured by Nikon Ti-S microscope equipped with a digital camera system (Nikon).

\subsection{Cell counting Kit-8 (CCK8) assay}

MHCC97L cells were plated in 96-well plates at the density of 1000 cells per well with $100 \mu \mathrm{L}$ of complete culture medium. After adhesion for $24 \mathrm{~h}$, the cells were cultured for additional 24, 48, 72, 96 and $120 \mathrm{~h}$. The wells only with the culture medium were used as blank controls. At each time point, the supernatant was removed and $100 \mu \mathrm{L}$ of DMEM medium containing $10 \mu \mathrm{L}$ of CCK8 (Dojindo, Japan) was added to each well for additional $3 \mathrm{~h}$ at $37^{\circ} \mathrm{C}$. The absorbance was recorded at $450 \mathrm{~nm}$. All experiments were independently repeated at least three times.

\subsection{Flow cytometry analysis}

To analyze cellular DNA content by flow cytometry, $1 \times 10^{6}$ cells were collected, rinsed and fixed overnight with $75 \%$ cold ethanol at $-20^{\circ} \mathrm{C}$. Cells were then treated with $100 \mu \mathrm{g}$ $\mathrm{mL}^{-1}$ RNase A in Tris-HCl buffer ( $\left.\mathrm{pH} 7.4\right)$ and stained with $25 \mu \mathrm{g} \mathrm{mL}^{-1}$ propidium iodide (PI, Sigma-Aldrich, USA). Samples were then subjected to the analysis by flow cytometry (FACSCalibur, BD Biosciences, USA) using CellQuest Pro software (BD Biosciences). Ten thousand cells were acquired and analyzed for the DNA content.

\subsection{Migration assay}

The MHCC97L cells $\left(1.5 \times 10^{5}\right.$ cells per $\left.100 \mu \mathrm{L}\right)$ in serum and insulin-free DMEM with or without anti-VEGF antibody were seeded on $8-\mu \mathrm{m}$ pore transwell inserts (Corning Costar No. 3422; Corning, USA). After 24 h, cells on the upper side of the inserts were removed with a cotton swab. 
For evaluation of cells that migrated onto the lower surface, inserts were fixed in $4 \%$ paraformaldehyde for $15 \mathrm{~min}$ and dyed in $0.1 \%$ crystal violet for $45 \mathrm{~min}$. The transwell membranes were then removed, placed on a glass slide with the side containing cells facing up, overlaid with a cover slip (VWR ${ }^{\circledR}$ Micro Cover Glasses, Round, No. 1). Migrated cells were counted in five non-overlapping locations, which covered approximately $70 \%$ of the insert membrane growth area, using a Nikon Ti-S microscope equipped with a digital camera system (Nikon). The experiment was repeated at least three times.

\subsection{Western blot}

Protein extracts were quantified and separated by $10 \%$ SDS-polyacrylamide gel, and transferred to the Immobilon TMPVDF Transfer Membranes (Millipore Corporation, USA). After blocking with 5\% nonfat milk in PBS, membranes were immunoblotted with anti-Flag antibody (F1804, Sigma, Germany) together with anti- $\beta$-actin antibody (Merck, Germany) as the internal control. Then horseradish peroxidase (HRP)-linked secondary antibody (Cell Signaling, USA) was incubated for $1 \mathrm{~h}$ at room temperature. The signals were detected by ImmobilonTM Western Chemiluminscent HRP Substrate kit (Millipore Corporation).

\subsection{Animal experiments}

GFP-Luciferase-labeled MHCC97L cells $\left(3 \times 10^{6}\right)$ suspended in $40 \mu \mathrm{L}$ of DMEM/Matrigel (1:1) were orthotopically inoculated into the left hepatic lobe of six-week-old male $\mathrm{BALB} / \mathrm{c}$ nude mice under anesthesia, which were obtained from Shanghai SLAC Laboratory Animal Co. (China). Anesthetized mice were retroorbitally injected with $75 \mathrm{mg}$ $\mathrm{kg}^{-1}$ D-Luciferin (360202, CellCyto), and the photon fluxes (photons per second) emitted from tumor cells was monitored by bioluminescent images (BLI). Bioluminescence images were acquired with the IVIS Imaging System (Xenogen, USA). Analysis was performed with Living Image software (Xenogen) by measuring photon flux of the upper abdominal and thoracic region. Data were normalized to the signal obtained immediately after injection (day 0 ). At day 35, mice were sacrificed. All livers and lungs were dissected, fixed with phosphate-buffered neutral formalin, paraffin-embedded and cut in slices.

All animals were handled in strict accordance to the "Guide for the Care and Use of Laboratory Animals" and the "Principles for the Utilization and Care of Vertebrate Animals", and all animal work was approved by the Institutional Animal Care and Use Committee at the Shanghai Jiao Tong University School of Medicine.

\subsection{Statistical analysis}

The $P$ values for comparison between line-linked groups were obtained by student's $t$-test. All statistical tests were two-sided, and $P<0.05$ were considered to be statistically significant.

\section{Results}

\subsection{Cbx4 increases in vitro vessel formation in its SIMs-dependent manner}

As we previously mentioned [17], Cbx4 enhances hypoxia-incubated HCC cell lines including MHCC97L cells to produce VEGF, a master stimulator of angiogenesis and vascular permeability in cancer tissues [20]. Thus, we detected whether $\mathrm{Cbx} 4$ affects the in vitro vessel formation in the co-culture system of HUVEC and MHCC97L cells with or without ectopic stable expression of Flag-tagged wildtype Cbx4 (Cbx4-WT), Cbx4-CDM or Cbx4- $\Delta \mathrm{SIM} 1 / 2$ (Figure 1A), in which Cbx4-CDM mutant carries F11A/ W35L double-mutation in its chromodomain of $\mathrm{Cbx} 4$ and loses its polycomb function but still presents SUMO E3 ligase activity, while $\mathrm{Cbx} 4-\Delta \mathrm{SIM} 1 / 2$ mutant is absent of their two SIMs and lose SUMO E3 ligase activity [17]. In line with those seen in HCC cell line SMMC-7721 [17], overexpression of Cbx4-WT and Cbx4-CDM in MHCC97L cells also increased the vessel formation of HUVEC. However, such an effect could not be seen in the Cbx4- $\Delta$ SIM1/2 mutant-expressing cells (Figure 1B and C).

\subsection{Cbx4 promotes the migration of MHCC97L cells by increasing VEGF}

It has been known that VEGF-mediated signaling in cancer is not limited to angiogenesis, and also contributes to other key aspects of tumorigenesis such as tumor metastasis [20,21]. Therefore, we tested the potential effects of Cbx4 and its mutants on the migration of MHCC97L cells by transwell experiments. Our results showed that hypoxia but not normoxia increased migration of MHCC97L cells, which was significantly enhanced by overexpressions of Cbx4-WT and Cbx4-CDM but not Cbx4- $\Delta$ SIM1/2 (Figure 2 ), suggesting that $\mathrm{Cbx} 4$ promotes the hypoxia-induced migration of cancer cells, which is also dependent on its SIMs domains.

To determine whether the increased VEGF under hypoxia contributes to the enhanced migration of MHCC97L cell with stable ectopic Cbx4-WT and Cbx4-CDM expression, we treated these cells together with anti-VEGF antibody to neutralize VEGF under hypoxia and normoxia, followed by the transwell experiments. The results showed that anti-VEGF antibody almost completely inhibited the hypoxia-stimulated migration of MHCC97L cells in spite of the expressions of Cbx4-WT, Cbx4-CDM or Cbx4- $\Delta \mathrm{SIM} 1 / 2$ (Figure 2). All these results indicate that Cbx4-promoting migration of MHCC97L cells is mediated by the increased 
A

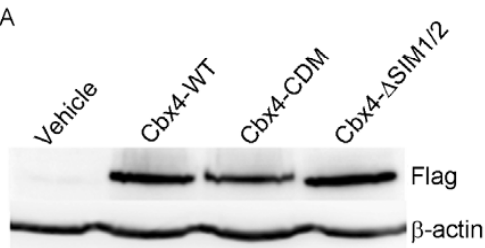

B

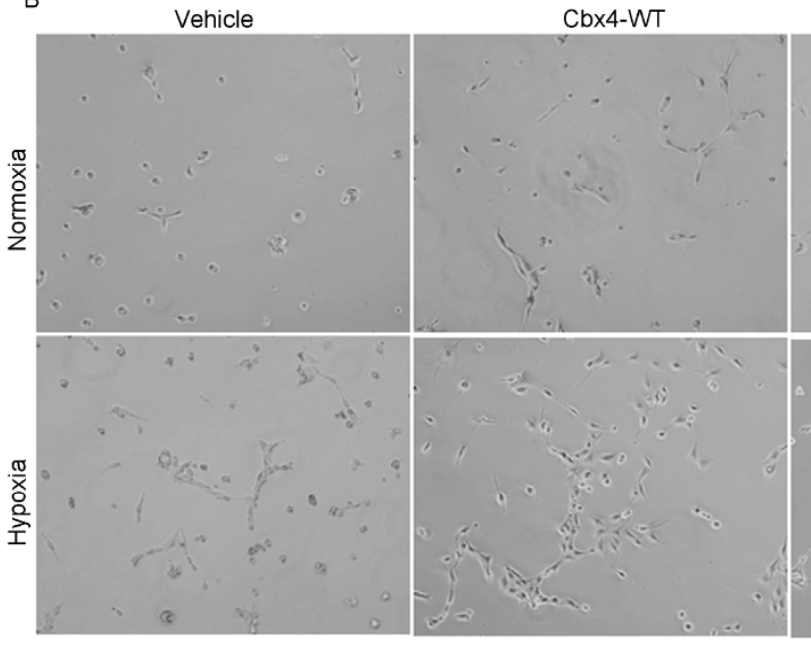

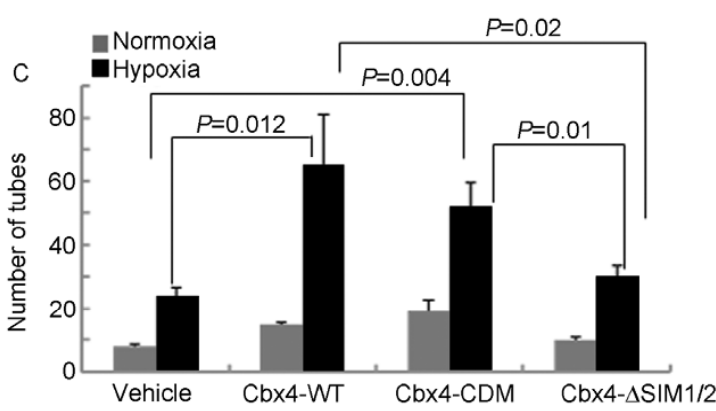

Cbx4-CDM

Cbx4- $\Delta \mathrm{SIM} 1 / 2$

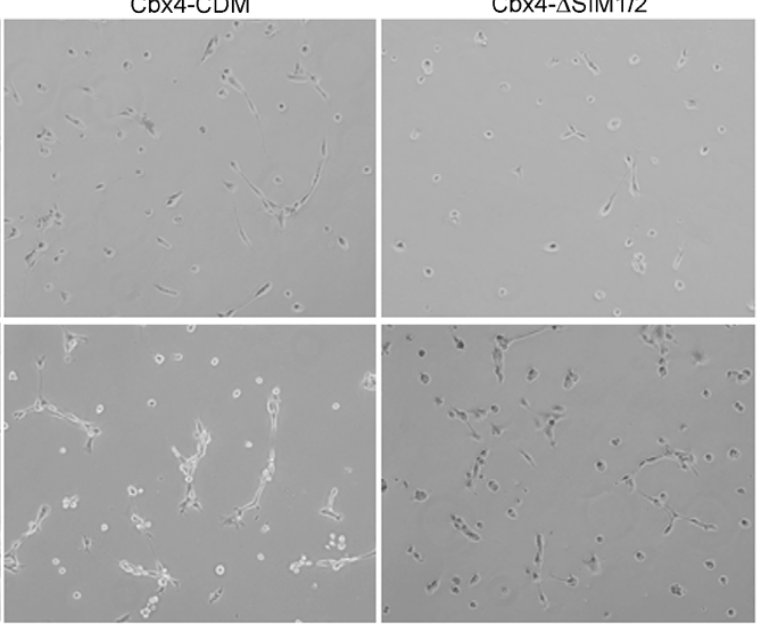

Figure $1 \mathrm{Cbx} 4$ overexpression in MHCC97L cells increases hypoxia-induced in vitro tube formation of HUVEC. A, Western blots for the indicated flag-tagged proteins with $\beta$-actin as a loading control in the lysates from MHCC97L cells ectopically expressing the vehicle or flag-tagged Cbx4-WT or its mutants. B, Photographs for the endothelial tubes in co-culture of MHCC97L cells stably expressing the flag-tagged genes as indicated together with HUVEC under normoxia or hypoxia for $16 \mathrm{~h}$. C, The number of the endothelial tubes formed by HUVEC in the co-culture assay, together with MHCC97L cells stably expressing the genes as indicated under normoxia or hypoxia for $16 \mathrm{~h}$. Data are mean \pm SD in triplicates in an independent experiment, which was repeated at least three times with the same results.

\section{VEGF expression.}

\subsection{Cbx4 fails to regulate growth and cell cycle pro- gression of MHCC97L cells}

Previously, we reported that $\mathrm{Cbx} 4$ did not affect cell growth of HCC cells under normoxia [17]. Here we further showed that ectopic expressions of $\mathrm{Cbx} 4$ and its two mutants did not significantly interfere with the growth of MHCC97L cells under hypoxia (Figure 3A). Furthermore, we analyzed the cell cycle distribution in these MHCC97L with or without overexpression of $\mathrm{Cbx} 4$ and its mutants. The results also support that $\mathrm{Cbx} 4$ and its mutants did not regulate cell cycle progression of the $\mathrm{HCC}$ cell line under either normoxia (Figure 3B) or hypoxia (Figure 3C).

\subsection{Cbx4 enhances the in vivo growth of MHCC97L cell-transplanted tumors}

Although $\mathrm{Cbx} 4$ expression did not impact the in vitro growth of MHCC97L cells, we still directly injected GFP-luciferase-labeled MHCC97L cells expressing vehicle,
Cbx4-WT, Cbx4-CDM and Cbx4- $\Delta$ SIM1/2 into the livers of $\mathrm{BALB} / \mathrm{c}$ nude mice, followed by monitoring luciferase photon fluxes each week. The results demonstrated that like those in SMMC-7721 cells [17], fluorescence intensities of tumors with ectopic expressions of Cbx4-WT and Cbx4CDM were greatly higher than those of vehicle and Cbx4$\Delta$ SIM1/2 (Figure 4A). The representative noninvasive BLI at the 5 th week after orthotopical injection are shown (Figure 4B). Then, we executed and dissected the nude mice in the 5th week post-transplantation. As depicted in Figure 4C, liver tumors with $\mathrm{Cbx} 4-\mathrm{WT}$ and $\mathrm{Cbx} 4-\mathrm{CDM}$ overexpressions were much bigger and more widely distributed in livers than those with vehicle and Cbx4- $\Delta$ SIM1/2-express- ing cells. In line with the notion, nude mice transplanted with Cbx4-WT and Cbx4-CDM but not Cbx4- $\Delta$ SIM1/2 had more numbers of tumor nodules in livers (Figure 4D). As consistent with our previous report in HCC cell line SMMC-7721 cells [17], additionally, Cbx4 and Cbx4-CDM but not Cbx4- $\Delta \mathrm{SIM} 1 / 2$ expressions remarkably increased VEGF expression in the transplanted tumors, as estimated by immunohistochemical staining (Figure 4E). 


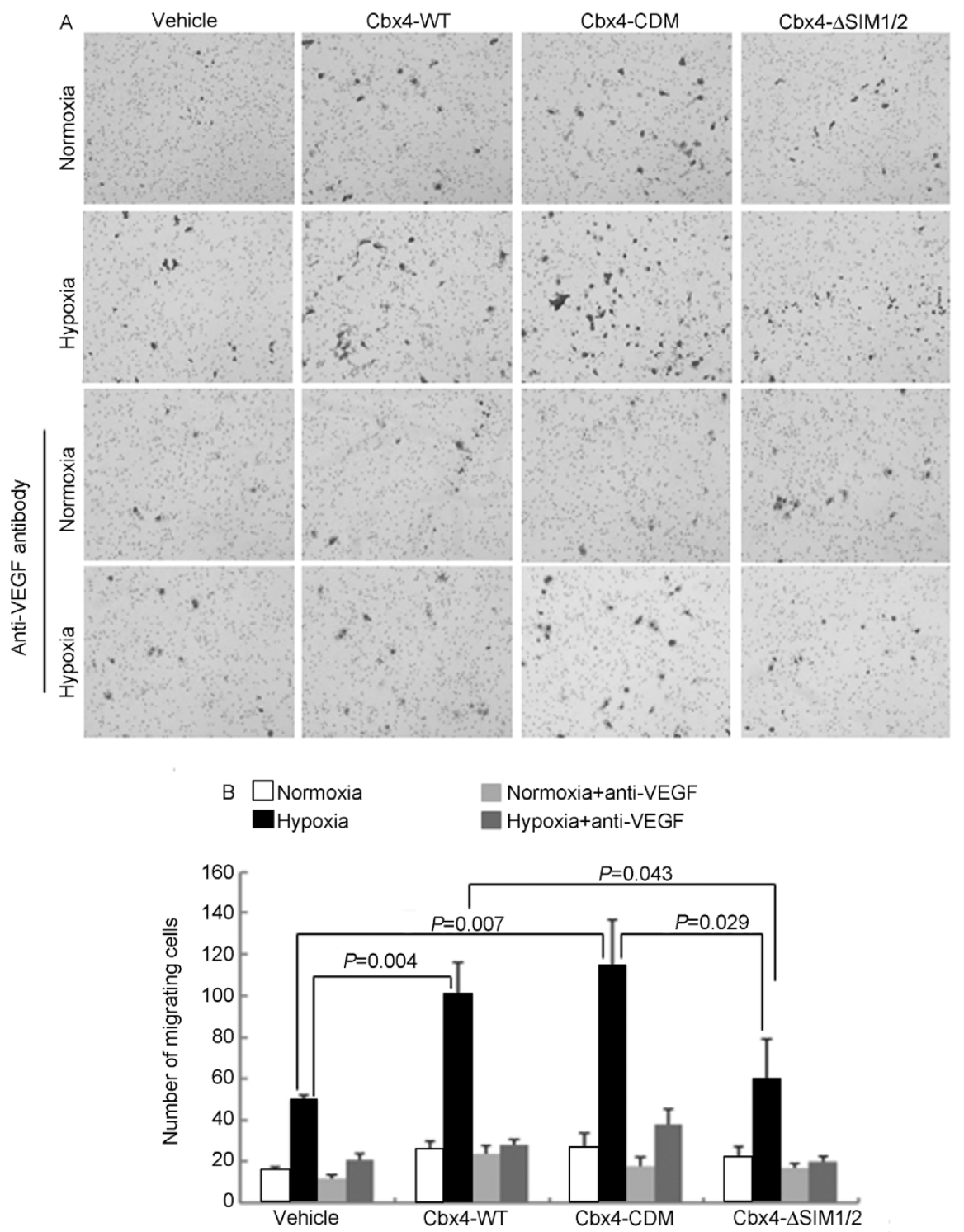

Figure $2 \mathrm{Cbx} 4$ promotes VEGF-mediated in vitro migration of MHCC97L cell. A, Images of cell migration were obtained by capturing migrated Flag-tagged Cbx4, its mutants or vehicle-infected MHCC $97 \mathrm{~L}$ cells on uncoated membranes of transwell chambers after $24 \mathrm{~h}$. B, Numbers of migrated cells were assessed by counting migrated MHCC97L cells on uncoated membranes. Data are mean \pm SD in triplicates in an independent experiment, which was repeated at least for three times with the same results.

\subsection{Cbx4 promotes the metastasis of MHCC97L cells in vivo}

Based on our observation that $\mathrm{Cbx} 4$ and $\mathrm{Cbx} 4-\mathrm{CDM}$ expression remarkably increased the in vitro migration of MHCC97L cells under hypoxia (Figure 2), we also observed the potential tumors in other tissues besides livers. The results showed that there was a higher frequency of pulmonary metastasis and the more tumor nodule numbers in lungs of the nude mice injected with MHCC97L cells stably expressed $\mathrm{Cbx} 4-\mathrm{WT}$ and $\mathrm{Cbx} 4-\mathrm{CDM}$ than vehicle and $\mathrm{Cbx} 4-\Delta \mathrm{SIM} 1 / 2$ (Figure $5 \mathrm{~A}$ ). A representative image for lung metastasis from nude mice with the transplantation of Cbx4-expressing MHCC97L cells is shown in Figure 5B.
All these results support that like SMMC7721 cells previously reported [17], Cbx4 but not its SUMO E3 ligase activity-absent $\mathrm{Cbx} 4-\Delta \mathrm{SIM} 1 / 2$ could promote in vivo metastasis of MHCC97L cells.

\section{Discussion}

Hepatocellular carcinoma is one of the most common cancers worldwide, with an increasing incidence in Asian and also in Western countries as a result of increasing hepatitis $\mathrm{B}$ and hepatitis C virus infection [22]. More than $80 \%$ of patients with HCC have been associated with cirrhosis and impaired liver function, making treatment of HCC more 

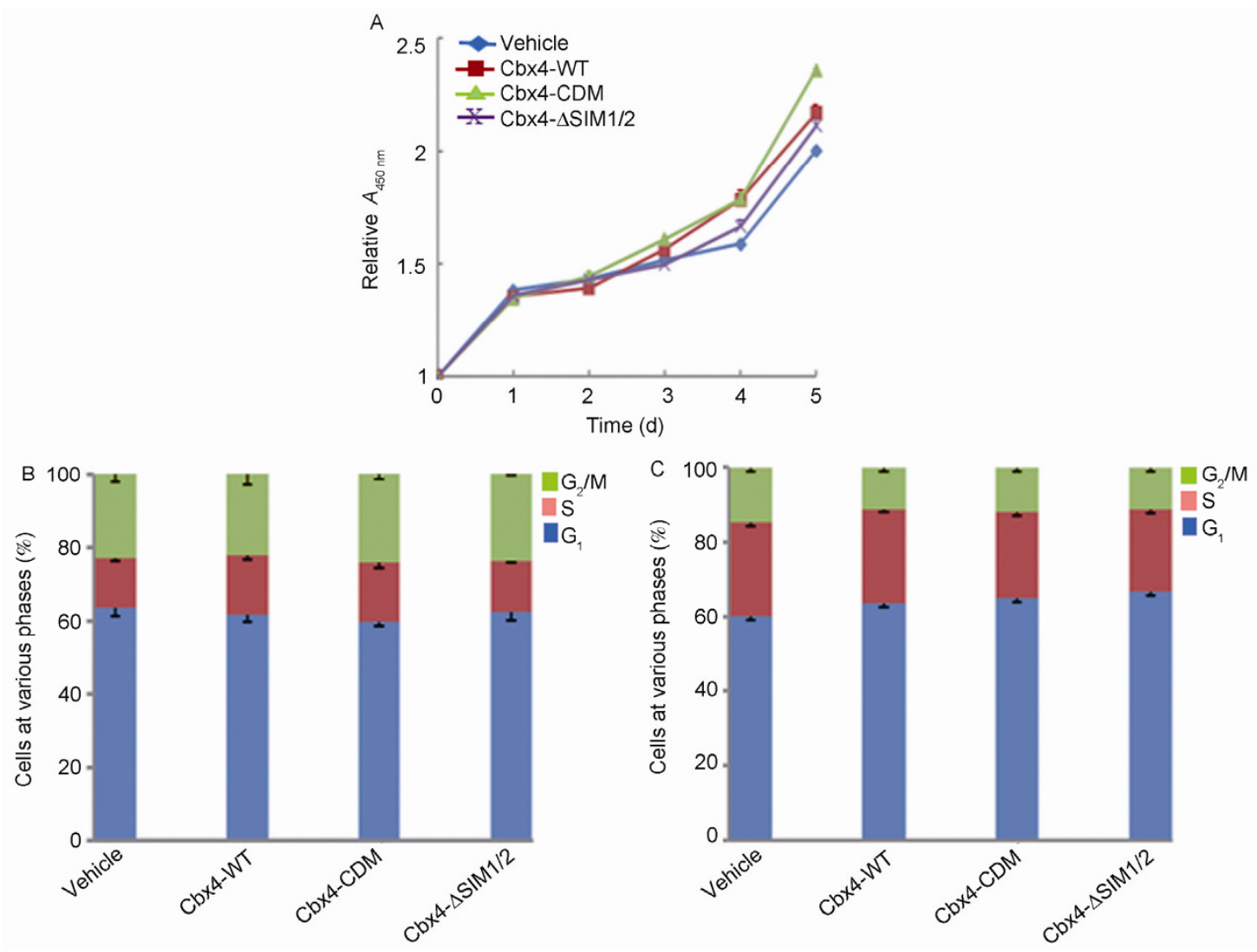

Figure $3 \mathrm{Cbx} 4$ has no effect on proliferation and cell cycle progression of MHCC97L cells. A, MHCC97L cells ectopically expressing Flag-tagged Cbx4-WT and its mutants were incubated under hypoxia for the indicated days. The growth rates were measured by CCK8 assay normalized to vehicle cells at $0 \mathrm{~h}$. B and C, MHCC97L cells transfected with Flag-tagged Cbx4-WT and its mutants were incubated under normoxia or hypoxia for $48 \mathrm{~h}$, followed by flow cytometric analysis for DNA distribution. The percentage of cells at each stage of cell cycle was quantified. All these experiments were repeated for at least three times with the same results.

difficult than many other cancers. HCC is a hypervascular tumor characterized by neovascularization, which plays an important role in the growth and progression of HCC. Therefore, it is proposed that angiogenesis provides a target for novel prognostic and therapeutic approaches to HCC $[23,24]$. Currently, available antiangiogenic cancer chemotherapy targeting the VEGF pathway by monoclonal anti-VEGF antibody bevacizumab or multi-targeted receptor tyrosine kinase inhibitors such as sorafenib has emerged $[25,26]$. On the other hand, HIF-1 induces expression of angiogenic growth factors such as VEGF, stromal derived factor 1 , angiopoietin 2, platelet-derived growth factor B and stem cell factor. Therefore, it is a key regulatory factor for angiogenesis in response to hypoxia and plays an important role in the pathogenesis of cancers including HCC, suggesting the possibility of HIF-1 $\alpha$ as a novel therapeutic target in HCC [27]. More recently, we reported that Cbx4 governs HIF-1 $\alpha$ to increase VEGF production and thus potentiate angiogenesis of HCC by its SUMO E3 ligase activity [17]. Because VEGF also contributes to other oncogenesis events besides angiogenesis [20,21,28], here we investigate the potential impacts of $\mathrm{Cbx} 4$ on migration and metastasis of HCC cell line MHCC97L. We demonstrate that the overexpression of $\mathrm{Cbx} 4$ but not its SUMO E3 ligase activity-absent mutant in MHCC97L increases in vitro hypoxia-stimulated vessel formation of HUVEC, which is consistent with our previous observation that $\mathrm{Cbx} 4$ enhances VEGF production in the HCC cell line SMMC-7721 [17]. More interestingly, we also find that $\mathrm{Cbx} 4$ promotes the migration on transwell of MHCC97L cells under hypoxia but not normoxia, for which its SIMs are necessary. Especially, anti-VEGF antibody almost completely inhibits migration-enhancing effect of $\mathrm{Cbx} 4$, indicating that this event is mediated by the increased VEGF expression.

It was reported that targeted disruption of $\mathrm{Cbx} 4$ causes severe hypoplasia of the fetal thymus as a result of reduced thymocyte proliferation [29]. $\mathrm{Cbx} 4$ was also shown to maintain human epidermal stem cells as slow-cycling and undifferentiated, and inhibit stem cell activation and differentiation through its SUMO ligase activity [30]. More recently, it was also reported that $\mathrm{Cbx} 4$ knockdown led to the decreased proliferation and impaired cell cycle progression in HCC cell lines [31]. However, our results show that Cbx4 fails to regulate growth and cell cycle progression of MHCC97L cells under either hypoxia or normoxia. In spite of this, tumors from subcutaneously transplanted 


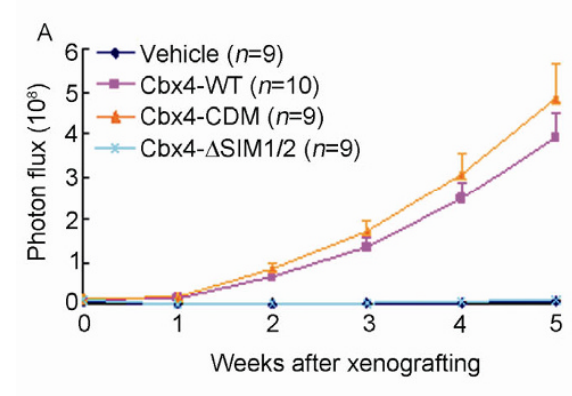

C
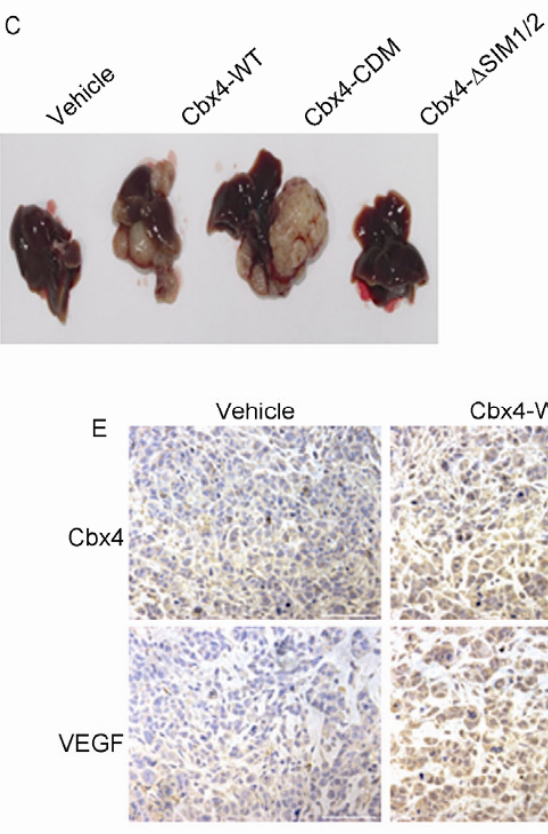

Cbx4-WT

D

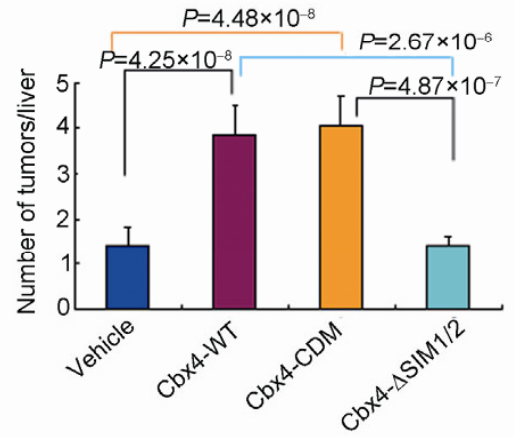

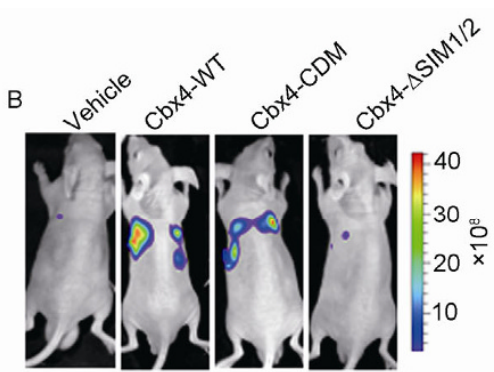

Cbx4-CDM

$\mathrm{Cbx} 4-\Delta \mathrm{SIM} 1 / 2$

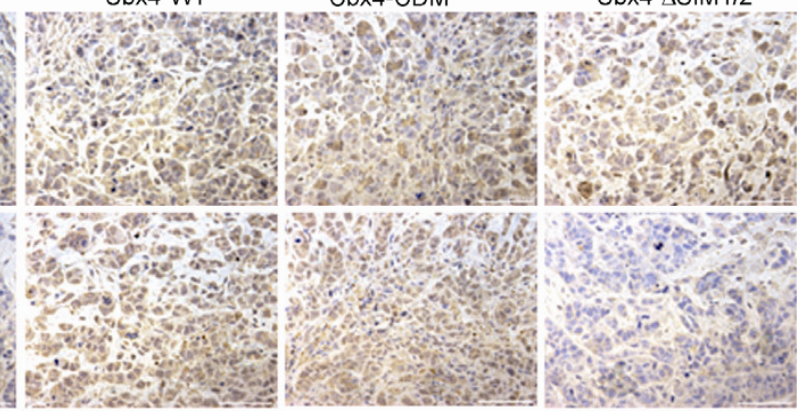

Figure 4 Cbx4 enhances in vivo progression of tumors injected with MHCC97L cells. GFP-luciferase-labeled Cbx4-WT and its mutants-infected MHCC97L cells were orthotopically injected into livers. A, The photon fluxes (photons per second) were monitored by bioluminescence imaging using Xenogen imaging system at the indicated weeks post injection. B, Four representative noninvasive BLI at day 35 after injection with the color scale depicting the photon fluxes emitted from the tumor cells. C, Four representative macroscopic observations of removed livers at day 35 post injection. D, The numbers of tumor nodules in the liver under microscope at day 35 post injection. E, The representative IHC staining images (scale bars, $100 \mu \mathrm{m}$ ) of Cbx 4 and VEGF in MHCC97L cells-originated tumors at day 35 post injection. Data are mean \pm SEM (A) or mean \pm SD (D) of mice in the numbers indicated in the parenthesis.
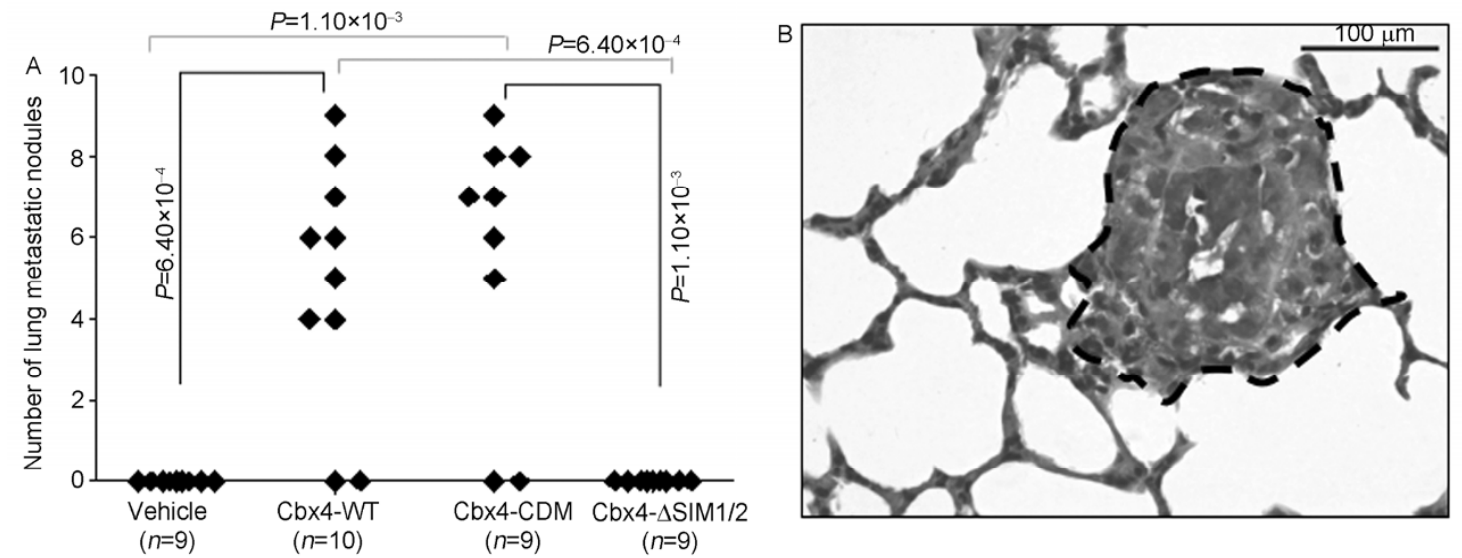

Figure 5 Cbx4 promotes in vivo metastasis of MHCC97L cells. A, The number of lung metastasis nodules under microscope at day 35 post injection. Data are mean $\pm \mathrm{SD}$ of mice in the number indicated in the parenthesis. $\mathrm{B}$, A representative hematoxylin and eosin staining image of lung metastasis nodule (rounding by black line) from Cbx4-expressing cells. 
SMMC7721 and MHCC97L cell lines with the overexpression of Cbx4-WT and CDM mutant but not $\Delta$ SIM1/2 mutant grew more rapidly compared with vehicle controls [17]. Here we further reveal that tumors from orthotopically transplanted MHCC97L cell lines with the overexpression of $\mathrm{Cbx} 4$ but not $\Delta \mathrm{SIM} 1 / 2$ mutant also grew more rapidly, as seen in orthotopically transplanted SMMC-7721 cells [17]. Of great importance, these orthotopically transplanted MHCC97L cells with $\mathrm{Cbx} 4$ overexpression present significantly higher ability of pulmonary metastasis.

Taken together, our results further support that $\mathrm{Cbx} 4$ plays an extremely important role in the process of progression and metastasis of HCC through its SUMO E3 ligase activity. Therefore, to explore the agents to target SUMO E3 ligase activity of $\mathrm{Cbx} 4$ might be of great significance for the treatment of HCC patients.

We appreciate Drs. Cheng JinKe (Shanghai Jiao Tong University School of Medicine), Qin LunXiu (Fudan University), Yi ZhengFang and Weng JieMin (East China Normal University) for providing us with cell lines or plasmids. This work was supported by the National Natural Science Foundation of China (91213304, 31101044), Shanghai Science and Technology Committee (11JC1406800), and Shanghai Committee of Education.

1 Schwartz YB, Pirrotta V. Polycomb silencing mechanisms and the management of genomic programmes. Nat Rev Genet, 2007, 8: 9-22

2 Morey L, Helin K. Polycomb group protein-mediated repression of transcription. Trends Biochem Sci, 2010, 35: 323-332

3 Di Croce L, Helin K. Transcriptional regulation by polycomb group proteins. Nat Struct Mol Biol, 2013, 20: 1147-1155

4 Su Y, Deng B, Xi R. Polycomb group genes in stem cell self-renewal: a double-edged sword. Epigenetics, 2011, 6: 16-19

5 Levine SS, King IF, Kingston RE. Division of labor in polycomb group repression. Trends Biochem Sci, 2004, 29: 478-485

$6 \mathrm{Au}$ SL, Ng IO, Wong CM. Epigenetic dysregulation in hepatocellular carcinoma: focus on polycomb group proteins. Front Med, 2013, 7: 231-241

7 Radulovic V, de Haan G, Klauke K. Polycomb-group proteins in hematopoietic stem cell regulation and hematopoietic neoplasms. Leukemia, 2013, 27: 523-533

8 Richly H, Aloia L, Di Croce L. Roles of the polycomb group proteins in stem cells and cancer. Cell Death Dis, 2011, 2: e204

9 Wotton D, Merrill JC. Pc2 and sumoylation. Biochem Soc Trans, 2007, 35: 1401-1404

10 Kagey $\mathrm{MH}$, Melhuish TA, Wotton D. The polycomb protein Pc2 is a SUMO E3. Cell, 2003, 113: 127-137

11 Long J, Zuo D, Park M. Pc2-mediated sumoylation of Smadinteracting protein 1 attenuates transcriptional repression of $\mathrm{E}$ cadherin. J Biol Chem, 2005, 280: 35477-35489

12 Li B, Zhou J, Liu P, Hu J, Jin H, Shimono Y, Takahashi M, Xu G. Polycomb protein $\mathrm{Cbx} 4$ promotes SUMO modification of de novo DNA methyltransferase Dnmt3a. Biochem J, 2007, 405: 369-378

13 Klein UR, Nigg EA. SUMO-dependent regulation of centrin-2. J Cell Sci, 2009, 122: 3312-3321
14 Roscic A, Moller A, Calzado MA, Renner F, Wimmer VC, Gresko E, Ludi KS, Schmitz ML. Phosphorylation-dependent control of Pc2 SUMO E3 ligase activity by its substrate protein HIPK2. Mol Cell, 2006, 24: 77-89

15 Fischle W, Wang Y, Jacobs SA, Kim Y, Allis CD, Khorasanizadeh S. Molecular basis for the discrimination of repressive methyl-lysine marks in histone H3 by Polycomb and HP1 chromodomains. Genes Dev, 2003, 17: 1870-1881

16 Merrill JC, Melhuish TA, Kagey MH, Yang SH, Sharrocks AD, Wotton D. A role for non-covalent SUMO interaction motifs in Pc2/Cbx4 E3 activity. PLoS ONE, 2010, 5: e8794

17 Li J, Xu Y, Long XD, Wang W, Jiao HK, Mei Z, Yin QQ, Ma LN, Zhou AW, Wang LS, Yao M, Xia Q, Chen GQ. Cbx4 governs HIF-1alpha to potentiate angiogenesis of hepatocellular carcinoma by its SUMO E3 ligase activity. Cancer Cell, 2014, 25: 118-131

18 Li J, Xu Y, Jiao HK, Wang W, Mei Z, Chen GQ. Regulation of hypoxia inducible factor-1alpha by SUMOylation and its significances in cancers. Sci China Life Sci, 2014, in press

19 Cui JF, Liu YK, Zhang LJ, Shen HL, Song HY, Dai Z, Yu YL, Zhang Y, Sun RX, Chen J, Tang ZY, Yang PY. Identification of metastasis candidate proteins among HCC cell lines by comparative proteome and biological function analysis of S100A4 in metastasis in vitro. Proteomics, 2006, 6: 5953-5961

20 Goel HL, Mercurio AM. VEGF targets the tumour cell. Nat Rev Cancer, 2013, 13: 871-882

21 Roberts E, Cossigny DA, Quan GM. The role of vascular endothelial growth factor in metastatic prostate cancer to the skeleton. Prostate Cancer, 2013, 2013: 418340

22 Lin S, Hoffmann K, Schemmer P. Treatment of hepatocellular carcinoma: a systematic review. Liver Cancer, 2012, 1: 144-158

23 Pang R, Poon RT. Angiogenesis and antiangiogenic therapy in hepatocellular carcinoma. Cancer Lett, 2006, 242: 151-167

24 Zhu AX, Duda DG, Sahani DV, Jain RK. HCC and angiogenesis: possible targets and future directions. Nat Rev Clin Oncol, 2011, 8: 292-301

25 Fang P, Hu JH, Cheng ZG, Liu ZF, Wang JL, Jiao SC. Efficacy and safety of bevacizumab for the treatment of advanced hepatocellular carcinoma: a systematic review of phase II trials. PLoS ONE, 2012, 7: e49717

26 Di Marco V, De Vita F, Koskinas J, Semela D, Toniutto P, Verslype C. Sorafenib: from literature to clinical practice. Ann Oncol, 2013, 24(Suppl 2): II30-37

27 Semenza GL. Defining the role of hypoxia-inducible factor 1 in cancer biology and therapeutics. Oncogene, 2010, 29: 625-634

28 Pugh CW, Ratcliffe PJ. Regulation of angiogenesis by hypoxia: role of the HIF system. Nat Med, 2003, 9: 677-684

29 Liu B, Liu YF, Du YR, Mardaryev AN, Yang W, Chen H, Xu ZM, $\mathrm{Xu}$ CQ, Zhang XR, Botchkarev VA, Zhang Y, Xu GL. Cbx4 regulates the proliferation of thymic epithelial cells and thymus function. Development, 2013, 140: 780-788

30 Luis NM, Morey L, Mejetta S, Pascual G, Janich P, Kuebler B, Cozutto L, Roma G, Nascimento E, Frye M, Di Croce L, Benitah SA. Regulation of human epidermal stem cell proliferation and senescence requires polycomb-dependent and -independent functions of Cbx4. Cell Stem Cell, 2011, 9: 233-246

31 Wang B, Tang J, Liao D, Wang G, Zhang M, Sang Y, Cao J, Wu Y, Zhang R, Li S, Ding W, Zhang G, Kang T. Chromobox homolog 4 is correlated with prognosis and tumor cell growth in hepatocellular carcinoma. Ann Surg Oncol, 2013, 20(Suppl 3): S684-692

Open Access This article is distributed under the terms of the Creative Commons Attribution License which permits any use, distribution, and reproduction in any medium, provided the original author(s) and source are credited. 